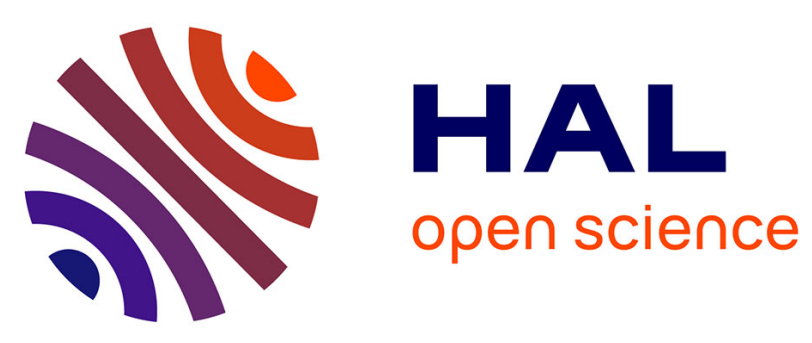

\title{
Analysis of and Fanconi Anemia genes in -negative Spanish breast cancer families
}

María J. García, Victoria Fernández, Ana Osorio, Alicia Barroso, Gemma

Llort, Conxi Lázaro, Ignacio Blanco, Trinidad Caldés, Miguel Hoya, Teresa Ramón y Cajal, et al.

\section{To cite this version:}

María J. García, Victoria Fernández, Ana Osorio, Alicia Barroso, Gemma Llort, et al.. Analysis of and Fanconi Anemia genes in -negative Spanish breast cancer families. Breast Cancer Research and Treatment, 2008, 113 (3), pp.545-551. 10.1007/s10549-008-9945-0 . hal-00478320

\section{HAL Id: hal-00478320 https://hal.science/hal-00478320}

Submitted on 30 Apr 2010

HAL is a multi-disciplinary open access archive for the deposit and dissemination of scientific research documents, whether they are published or not. The documents may come from teaching and research institutions in France or abroad, or from public or private research centers.
L'archive ouverte pluridisciplinaire HAL, est destinée au dépôt et à la diffusion de documents scientifiques de niveau recherche, publiés ou non, émanant des établissements d'enseignement et de recherche français ou étrangers, des laboratoires publics ou privés. 


\title{
Analysis of $F A N C B$ and $F A N C N / P A L B 2$ Fanconi Anemia genes in $B R C A 1 / 2$-negative Spanish breast cancer families
}

\author{
María J. García · Victoria Fernández · Ana Osorio · Alicia Barroso · \\ Gemma LLort · Conxi Lázaro • Ignacio Blanco • Trinidad Caldés • \\ Miguel de la Hoya · Teresa Ramón y Cajal · Carmen Alonso • \\ María-Isabel Tejada · Carlos San Román · Luis Robles-Díaz • \\ Miguel Urioste $\cdot$ Javier Benítez
}

Received: 12 February 2008/ Accepted: 12 February 2008/Published online: 27 February 2008

(C) Springer Science+Business Media, LLC. 2008

\begin{abstract}
Recent reports have shown that mutations in the FANCJ/BRIP1 and FANCN/PALB2 Fanconi Anemia (FA) genes confer a moderate breast cancer risk. Discussion has been raised on the phenotypic characteristics of the PALB2-associated families and tumors. The role of $F A N C B$ in breast cancer susceptibility has not been tested to date. Likewise PALB2 mutation frequency has not been studied in Spanish population. We analyzed the complete coding sequence and splicing sites of $F A N C B$ and PALB2 in 95 index cases of $B R C A 1 / 2$-negative Spanish breast cancer families. We also performed an exhaustive screening of three previously described rare but recurrent PALB2 mutations in 725 additional probands. Pathogenic changes
\end{abstract}

Electronic supplementary material The online version of this article (doi:10.1007/s10549-008-9945-0) contains supplementary material, which is available to authorized users.

\footnotetext{
M. J. García · V. Fernández · A. Osorio · A. Barroso ·

M. Urioste · J. Benítez ( $\square)$

Group of Human Genetics, Human Cancer Genetics Program,

Spanish National Cancer Centre (CNIO), C/ Melchor Fernández

Almagro 3, 28029 Madrid, Spain

e-mail: jbenitez@cnio.es
}

M. J. García · J. Benítez

Centro de Investigación Biomédica en Red de Enfermedades

Raras (CIBERER), Instituto de Salud Carlos III, Madrid, Spain

G. LLort · I. Blanco

Genetic Counseling Unit, Prevention and Cancer Control

Department, Catalan Institute of Oncology (ICO),

08907 L'Hospitalet, Barcelona, Spain

\section{Lázaro}

Translational Research Laboratory, Catalan Institute of

Oncology (ICO), 08907 L'Hospitalet, Barcelona, Spain were not detected in FANCB. We found a novel PALB2 truncating mutation c.1056_1057delGA (p.K353IfsX7) in one of the 95 screened patients, accounting for a mutation frequency of $1 \%$ in our series. Further comprehensive screening of the novel mutation and of previously reported rare but recurrent $P A L B 2$ mutations did not reveal any carrier patient. We report the first example of $\mathrm{LOH}$ occurring in a $P A L B 2$-associated tumor. Our results rule out a major contribution of $F A N C B$ to hereditary breast cancer. Our data are consistent with the notion of individually rare $P A L B 2$ mutations, lack of mutational hotspots in the gene and existence of between-population disease-allele heterogeneity. We show evidence that PALB2 loss of function might also conform to the inactivation model of a classic tumor-suppressor gene and present data that adds to the clinically relevant discussion about the existence of a $P A L B 2$-breast cancer phenotype.

T. Caldés · M. de la Hoya

Laboratory of Molecular Oncology, San Carlos University

Hospital, 28040 Madrid, Spain

T. Ramón y Cajal · C. Alonso

Service of Medical Oncology, La Santa Creu i Sant Pau

Hospital, 08025 Barcelona, Spain

M.-I. Tejada

Molecular Genetics Laboratory, Cruces Hospital,

48903 Baracaldo, Bilbao, Spain

C. San Román

Genetics Department, Ramón y Cajal Hospital,

28034 Madrid, Spain

L. Robles-Díaz

Oncology Department, Doce de Octubre Hospital,

28041 Madrid, Spain 
Keywords FANCN/PALB2 $F A N C B \cdot$ Fanconi Anemia Genotypic-phenotypic correlation .

Hereditary breast cancer $\cdot$ PALB2 tumor

\section{Introduction}

Fanconi Anemia (FA) is a rare recessive disorder (heterozygote frequency of around 1 in 250-300) characterized by chromosomal fragility, progressive bone marrow failure, congenital abnormalities and cancer predisposition including leukemias and solid tumors [1]. To date 13 FA complementation groups (FA-A, B, C, D1, D2, E, F, G, I, J, $\mathrm{L}, \mathrm{M}$, and $\mathrm{N}$ ) have been described and with the recent identification of the gene responsible for subtype I [2] all 13 genes associated with the complementation groups are currently known. FA-A is the most common FA subtype accounting for approximately $60 \%$ of cases worldwide. The second most common groups are FA-C and FA-G with frequencies close to $9-10 \%$, all remaining subtypes showing individual incidences lower that 4\% [3]. However, these general frequencies may show variation in specific populations related to the existence of founder mutations as it has been described in Ashkenazy Jewish or Spanish Gypsies [4, 5]. Eight FA proteins, FANCA, B, C, E, F, G, L and $\mathrm{M}$ form a complex (FA nuclear core complex) that activates FANCD2 and, as recently shown, also the newly discovered FANCI protein through monoubiquitination [2, 6]. FANCD2 and FANCI interact with each other to form what it has been called the FA "ID" complex, both proteins being interdependent for their respective monoubiquitination [2]. Upon activation, the ID complex translocates to sites of DNA damage in chromatin, where interacts with the downstream FA proteins (acting downstream the ubiquitination step) and/or other proteins participating in recognition and repair of DNA damage [6].

In 2002 Howlett et al. discovered that the gene responsible for FA-D1 complementation group was BRCA2, which was the key fact that linked breast cancer susceptibility and the Fanconi Anemia-DNA repair pathway [7]. Subsequent works considered plausible that heterozygous mutation in FA genes other than FANCD1/BRCA2 could be responsible for and increased susceptibility to breast cancer and evaluated such hypothesis. In this line of research, FANCA, FANCC, FANCD2, FANCE, FANCF and FANCG were screened for mutations in familial breast cancer patients negative for mutations in the BRCA1 and BRCA2 genes [8] but pathogenic sequence variants were not found. Similar analysis of FANCJ/BRIPl was undertaken by several groups [9-12], the gene being recently reported as a breast cancer-moderate risk gene [13].

Recent data indicates that heterozygous mutations in PALB2 ("Partner and Localizer of BRCA2") -the gene found to be responsible for the most recently described FA-N complementation group [14, 15]- account for a proportion of around $1 \%$ of hereditary breast cancer non due to BRCA1/2 mutations [16, 17]. The relative risk of breast cancer associated with this gene was estimated at 2.3 [16], value that increased to approximately 4-fold in a study carried out in Finish population where a founder mutation was detected [18]. Initial screening of the gene in breast cancer families from other specific ethnic backgrounds such as Ashkenazi Jewish or French Canadian population did not reveal any additional $P A L B 2$ founder mutations [17]. However, a very recent extended study of French Canadian population allowed the identification of a novel PALB2 founder mutation [19]. Finnish and Canadian studies included loss of heterozygosity ( $\mathrm{LOH})$ and immunohistochemical analysis of $P A L B 2$-associated breast tumors. Early reports shown that most $P A L B 2$-neoplasms were oestrogen and progesterone receptor-positive, sharing phenotypic characteristic of $B R C A 2$ tumors. In contrast, the latest Canadian study suggests that these initial phenotypic observations might not be as universal as formerly thought. None of the PALB2 tumors reported to date have been found to present $\mathrm{LOH}$, which has raised speculation on alternative mechanisms of $P A L B 2$ functional inactivation.

In view of these antecedents we have analyzed the entire coding sequence and intron-exon boundaries of $F A N C B$ and $P A L B 2$ in $95 B R C A 1 / 2$-negative Spanish breast cancer families. Implication of heterozygous mutations of $F A N C B$ in breast cancer predisposition has not been tested to date. Similarly, there is no data on $P A L B 2$ mutation frequency in Spanish families of hereditary breast cancer. Our analysis has also encompassed a thorough screening of three previously reported rare but recurrent PALB2 mutations, c.3113G $>$ A (p. W1038X), c.3116delA (p. N1039IfsX2) and c.3549C $>\mathrm{G}$ (p.Y1183X) [16], in 725 additional $B R C A 1 / 2$-negative patients.

\section{Materials and methods}

Patients and controls

Index cases from 95 BRCA1/BRCA2 mutation-negative Spanish breast and ovarian cancer families were screened for mutations within the entire coding sequence and splicing sites of $F A N C B$ and $P A L B 2$ genes. An additional set of 725 index cases from $B R C A 1 / B R C A 2$ mutationnegative families were specifically screened for the previously reported $P A L B 2$ recurrent mutations c. $3113 \mathrm{G}>\mathrm{A}$ (p. W1038X), c.3116delA (p. N1039IfsX2) and c.3549C $>$ G (p.Y1183X) [16] as well as for novel mutations and/or interesting sequence variants found in the initial set of 95 patients. Families were ascertained in different Spanish 
hospitals and were selected for mutation analysis if they contained either (i) at least three cases of breast or ovarian cancer in the same family line; or (ii) at least two firstdegree relatives diagnosed with breast cancer before age 50 ; or (iii) at least one case of breast cancer and one case of ovarian or bilateral breast cancer in the same family line; or (iv) at least one case of breast and ovarian cancer; or (v) at least one case of male breast cancer. Controls consisted of 760 healthy individuals representative of the Spanish population, mainly recruited from the Menopause Research Centre at the Instituto Palacios (Madrid, Spain) and from the College of Lawyers (Madrid, Spain). Details of this control series have been previously published [20]. The necessary ethics committee approval was obtained as well as informed consent from all participants in the study.

\section{Analysis of BRCA1 and BRCA2}

All index cases had been previously screened for mutations in the BRCA1/2 genes by Denaturing High Performance Liquid Chromatography (DHPLC) and/or other comprehensive approaches described in detail elsewhere [21-25] and found to be negative.

\section{Mutation analysis of $F A N C B$ and $P A L B 2$ genes}

DNA from the index cases was subjected to Whole Genome Amplification (WGA) by using the GenomiPhi V2 Amplification Kit (GE Healthcare UK Limited, Buckinghamshire, UK) according to the manufacturerś instructions. Then genomic fragments covering $F A N C B$ and PALB2 exons and splicing sites were PCR-amplified. Primers were designed to generate amplicons of size up to $350 \mathrm{bp}$ to ensure optimal yield from WGA-DNA. In all, 17 and 24 PCR fragments were required to cover $F A N C B$ and $P A L B 2$, respectively. Seven primer pairs spanning exons 6-12 of $P A L B 2$ were taken from a previous study [15], those reported but generating fragments larger than $350 \mathrm{bp}$ being discarded and redesigned. All primer pairs are detailed in Supplementary Table 1. PCR-products were analyzed by DHPLC on the WAVE HT system (Transgenomic, Omaha, $\mathrm{NE}$ ) using an acetonitrile gradient and scrutinized for aberrant profiles with the Navigator ${ }^{\text {TM }}$ Software (Transgenomic, Omaha, NE). Samples representative of each DHPLC pattern were bi-directionally sequenced using the BigDyeTerminator Cycle sequencing kit and a 3730 automated sequencer (ABI Perkin Elmer). All non-described variants/mutations were confirmed by sequencing a fresh aliquot of the non-WGA stock DNA.

Extended screening of previously described PALB2 recurrent mutations, new mutations, and interesting sequence variants in 725 additional index cases was performed by the KBiosciences (Herts, UK) fluorescence-based competitive allele-specific PCR assay (KASPar) except for those changes consisting of deletions that were analyzed by DHPLC. Details of the KASPar methodology can be found at http://www.kbioscience.co.uk/. Analysis of the PALB2 recurrent mutations c. $3113 \mathrm{G}>\mathrm{A}$, c. $3116 \mathrm{delA}$ and c. $3549 \mathrm{C}>\mathrm{G}$ included positive controls kindly provided by Prof. N. Rahman. Fisher exact test was used to test differences in allele frequencies when comparison with control individuals was performed (PALB2 sequence variant c.629C $>\mathrm{T}$, p.P210L).

GenBank Reference sequences used for naming sequence variants were NM_152633.2 and NT_011757.15 for FANCB and NM_024675.3 and NT_010393.15 for $P A L B 2$. Standardized nomenclature was reported considering the A of the ATG initiation codon of the coding DNA Reference Sequence as nucleotide position $+1[26,27]$.

Loss of heterozygosity (LOH) analysis

Sections from paraffin-embedded tumor from PALB2 positive patient were obtained following revision by a pathologist and subsequent macrodissection in order to enrich sample with pure cancer cell population. DNA was extracted by standard proteinase $\mathrm{K}$ protocol after desparaffination. PALB2 sequence segment spanning the gene mutation was amplified by PCR (using a reduced number of cycles) and sequenced. DNA from peripheral blood of the patient was amplified and sequenced simultaneously along the procedure. LOH was scored by a significant reduction of at least $30 \%$ of peak height in the wild allele relative to normal sequence trace.

\section{Results}

FANCB mutation analysis

In order to establish the possible role of $F A N C B$ as a breast cancer susceptibility gene, 95 index cases from breast cancer families negative for $B R C A 1$ and $B R C A 2$ mutations were screened for sequence variants. Analysis of $F A N C B$ complete coding sequence and splicing regions did not reveal any pathogenic mutation. Four sequence variants were identified, three of them were non-coding (located at the $5^{\prime}$ upstream region or introns) and one was exonic (Table 1). All had been previously reported except for one of the intronic variants $(c .2165+8 \mathrm{~A}>\mathrm{G})$ that did not involved a consensus splice site and was not additionally investigated.

PALB2 mutation analysis

We analyzed the complete coding sequence and splicing sites of $P A L B 2$ in 95 probands from breast cancer families 
Table $1 F A N C B$ and $P A L B 2$ sequence variants identified in Spanish familial breast cancer patients

\begin{tabular}{|c|c|c|c|c|}
\hline Gene/location & Nucleotide change & Protein change & Heterozygote frequency $\%(\mathrm{n} / \mathrm{N})^{\mathrm{a}}$ & Reference $^{\mathrm{b}}$ \\
\hline \multicolumn{5}{|l|}{$F A N C B$} \\
\hline \multicolumn{5}{|l|}{ Coding } \\
\hline Ex 3 & c. $1004 \mathrm{G}>\mathrm{A}$ & p.G335E & $17(16 / 94)$ & rs.41309679 \\
\hline \multicolumn{5}{|l|}{ Non-coding } \\
\hline $5^{\prime}$ flanking & c. $-221 \mathrm{C}>\mathrm{A}$ & - & $43(40 / 93)$ & rs. 2285490 \\
\hline IVS5 & c. $1427-10 \mathrm{~T}>\mathrm{C}$ & - & $46(42 / 92)$ & rs. 2905223 \\
\hline IVS8 & c. $2165+8 \mathrm{~A}>\mathrm{G}$ & - & $1(1 / 89)$ & - \\
\hline \multicolumn{5}{|l|}{$P A L B 2$} \\
\hline \multicolumn{5}{|l|}{ Coding } \\
\hline \multirow[t]{8}{*}{ Ex 4} & c. $629 \mathrm{C}>\mathrm{T}$ & p.P210L & $0.37(3 / 794)$ & 1 \\
\hline & c. $765 \mathrm{~T}>\mathrm{C}$ & p.D255D & $1(1 / 93)$ & 1 \\
\hline & c. $999 \mathrm{C}>\mathrm{T}$ & p.T333T & $0.12(1 / 797)^{\mathrm{d}}$ & - \\
\hline & c. $1010 \mathrm{~T}>\mathrm{C}$ & p.L337S & $5(42 / 797)^{\mathrm{d}}$ & 1,2 \\
\hline & c.1056_1057delGA ${ }^{\mathrm{c}}$ & p.K353IfsX7 & $0.12(1 / 797)$ & 1 \\
\hline & c. $1194 \mathrm{G}>\mathrm{A}$ & p.V398V & $0.12(1 / 797)^{\mathrm{d}}$ & 1 \\
\hline & c. $1572 \mathrm{~A}>\mathrm{G}$ & p.S524S & $1(1 / 95)$ & 1 \\
\hline & c. $1676 \mathrm{~A}>\mathrm{G}$ & p.Q559R & $22(21 / 95)$ & rs. 152451 \\
\hline Ex 5 & c. $2014 \mathrm{G}>\mathrm{C}$ & p.E672Q & $5(5 / 95)$ & 1 \\
\hline Ex 7 & c. $2590 \mathrm{C}>\mathrm{T}$ & p.P864S & $5(5 / 95)$ & 1 \\
\hline \multirow[t]{2}{*}{ Ex 8} & c. $2794 \mathrm{G}>\mathrm{A}$ & p.V932M & $1(1 / 95)$ & 1,2 \\
\hline & c. $2816 \mathrm{~T}>\mathrm{G}$ & p.L939W & $1(1 / 95)$ & 1 \\
\hline Ex 9 & c. $2993 \mathrm{G}>\mathrm{A}$ & p.G998E & $3(3 / 91)$ & 1 \\
\hline Ex 12 & c. $3300 \mathrm{~T}>\mathrm{G}$ & p.T1100T & - & 1,2 \\
\hline \multicolumn{5}{|l|}{ Non-Coding } \\
\hline 5'ÚTR & c. $-47 \mathrm{G}>\mathrm{A}$ & - & $4(4 / 94)$ & rs. 8053188 \\
\hline IVS3 & c. $212-58 \mathrm{~A}>\mathrm{C}$ & - & $5(5 / 95)$ & - \\
\hline IVS4 & c. $1684+29 A>G$ & - & $2(2 / 95)$ & - \\
\hline IVS6 & c. $2586+31 \mathrm{~T}>\mathrm{G}$ & - & $1(1 / 95)$ & - \\
\hline IVS6 & c. $2587-38 \mathrm{C}>\mathrm{G}$ & - & $1(1 / 95)$ & - \\
\hline
\end{tabular}

GenBank Reference sequences used were NM_152633.2 and NT_011757.15 for FANCB and NM_024675.3 and NT_010393.15 for PALB2. Nucleotide position +1 corresponds to the A of the ATG translation initiation site of the coding DNA Reference Sequences. ${ }^{a}$ Frequency refers to number of carriers (n) out of successfully screened samples (N). Screened samples were 95 for all fragments except for that containing the novel mutation and sequence variant c.629C $>$ T where 725 additional cases were tested. ${ }^{\mathrm{b}}$ dbSNP identifier or previous studies reporting the sequence variants are indicated: 1, Rahman et al. 2007 [16]; Erkko et al, 2007 [18]. ${ }^{\mathrm{c}}$ Novel mutation. ${ }^{\mathrm{d}}$ Frequency of sequence variants contained within same PCR-fragment as the novel mutation

negative for mutations in $B R C A 1$ and $B R C A 2$ genes aiming to define the mutation frequency of the gene in Spanish population. Sequencing of one index case from our families revealed a non-previously reported frameshift mutation, c.1056_1057delGA (p.K353IfsX7), which is predicted to generate a translation-stop seven codons downstream from the first affected amino acid. Extended analysis of this novel mutation by DHPLC in 725 additional BRCA1/2negative probands, 702 of them successfully screened, did not show any other carrier of the mutation. The pedigree of the family of the $P A L B 2$-mutation carrier is presented in Fig. 1. Interestingly, the pedigree corresponds to a female and male breast cancer family. Index case developed breast cancer at age 54, her father and one of her two sisters having also developed breast cancer at ages 78 and 49, respectively. Unfortunately we could not assess the mutation carrier status of any of the proband's relatives with breast cancer to evaluate penetrance. However, paraffinembedded tissue from the proband's tumor was available and $\mathrm{LOH}$ and immunohistochemical analysis were performed (shown below).

In addition to the c.1056_1057delGA (p.K353IfsX7) truncating mutation we identified 18 PALB2 sequence variants (Table 1). Five of the variants were non-coding and thirteen coding. Of the non-coding variants one was located at the $5^{\prime}$ UTR region and had already been reported and four corresponded to non-previously described intronic variants distant from the intron-exon boundaries not likely 


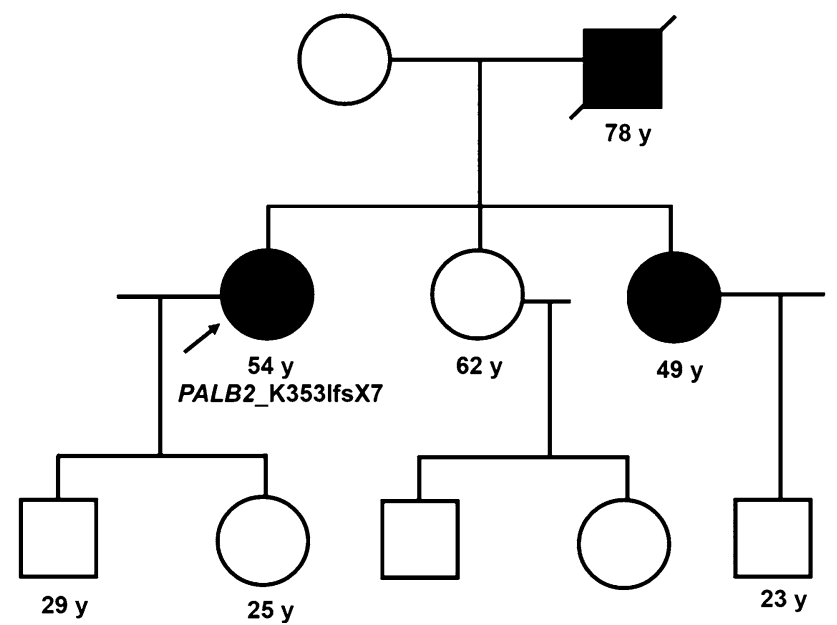

Fig. 1 Pedigree of the breast cancer family carrying the c.1056 1057delGA (p.K353IfsX7) PALB2 mutation. The proband screened for $P A L B 2$ mutations is indicated by an arrow. Segregation analysis was not possible because of lack of DNA from family members other than the index case. Individuals with breast cancer are marked with a black circle/square. Age at diagnosis for cancer patients and at monitoring for healthy individuals is shown when known. A slashed circle/square indicates a deceased individual

\section{A Peripheral blood}

Wt. A A AC AG AGA A A T C T T T A A A T C T C C C A

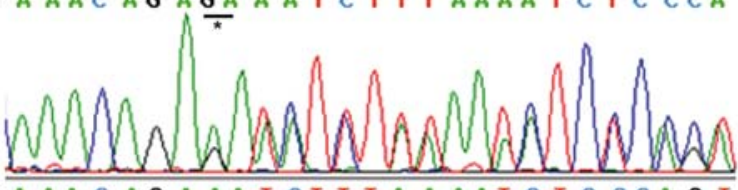

Mut. A A A C A A A A TCT T TA A A ATCTC CCA G T

\section{B Tumour}

Wt. A A A C A G A G A A T CTTTAAAA TCTCCCA

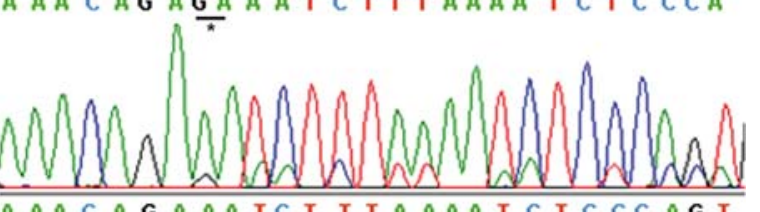

Mut. A A A C A G A A A TC T T T A A A A T C T C C C A G T

Fig. 2 Sequence chromatograms corresponding to the c.1056_ 1057delGA (p.K353IfsX7) PALB2 mutation carrier. Wt: Wild-type allele strand. Mut: Mutant allele strand. Asterisk indicates deleted GA and frameshift starting point. (a) Sequence from the patient's peripheral blood. Overlapping peaks of wild type and mutant strand show similar signal intensity. (b) Chromatogram the patient's macrodissected paraffin-embedded tumor. Wild-type strand shows a clearly diminished signal compared to that of the mutant strand indicating $\mathrm{LOH}$ of the wild-type allele. DNA from peripheral blood and tumor were amplified simultaneously under same PCR conditions

to be pathogenic. Of the coding variants five were synonymous and eight non-synonymous, all of them previously reported [16, 18] except for the synonymous variant c.999C $>$ T (p.T333T). The missense variant c.629C $>\mathrm{T}$ (p.P210L) was detected in 2 out of 95 index cases (2\%), while none out of 923 probands and 1 out of 1084 controls previously analyzed by Rahman et al. presented it [16].
Taking into account that in silico predictions suggest that this change is likely to affect the protein function [16], we decided to further characterize this change in our population in a case-control study. KASPar assay detected one carrier of the variant both, in our set of additional BRCAl/ 2 -negative patients ( $n=699$ successfully genotyped) and in our group of controls ( $n=735$ successfully genotyped). Our results taken together with those of Rahman et al. render global frequencies of $0.17 \%$ in cases (3/1717) and $0.10 \%$ in controls $(2 / 1819)(P=0.7)$, which supports a neutral role for this missense variant.

Rahman et al. reported three PALB2 mutations that appeared in more than one proband out of 923 screenedhereditary breast cancer families, the probands with identical mutations being unrelated and from different parts of the UK. None of these previously reported rare but recurrent mutations, c.3113G $>$ A (2/923), c.3116delA (3/923) and c.3549C $>\mathrm{G}$ (3/923), were detected either in our initial set of $95 B R C A 1 / 2$-negative cases or in the subsequently screened 725 additional index cases. Positive controls included along with our samples allowed us to ensure the adequacy of our screening methods to discriminate these mutations.

\section{$P A L B 2$ tumor features}

In order to obtain information about the clinicopathological characteristics of the PALB2 tumor and to assess whether loss of heterozygosity had occurred we requested the Pathology report and paraffin block of the breast tumor from the mutation-positive patient. The tumor was a grade III-invasive ductal carcinoma with areas of apocrine carcinoma in situ negative for oestrogen and progesterone receptors. We also assessed HER2 protein expression by the DAKO HercepTest ${ }^{\mathrm{TM}}$ and score protocol system (DAKO A/S, Glastrup, Denmark) that indicated a negative status. P53 staining was low $(<10 \%)$ while proliferative index measured by Ki-67 showed a value of $30 \%$. To test whether LOH had occurred in the PALB2-associated tumor we PCR-amplified and sequenced the segment spanning the c.1056_1057delGA (p.K353IfsX7) mutation from DNA obtained from the paraffin-embedded tissue (along with DNA from the peripheral blood of the patient as control). Chromatogram of the tumor showed a height reduction greater than $30 \%$ in the peaks from the wild type-strand relative to the sequence trace of the mutant allele indicating LOH (Fig. 2).

\section{Discussion}

FANCD1/BRCA2 is one of the two currently known highpenetrance breast cancer susceptibility genes [7]. Two 
other FA genes, FANCN/PALB2 and FANCJ/BRIP1, have been recently shown to confer a moderate risk to develop breast cancer [13, 16, 28]. These recent findings have further strengthened the link between the FA proteins and breast cancer but while most FA genes have been tested for their association with breast cancer [8], there are still a few whose implications in breast cancer susceptibility remains unknown. $F A N C B$ is one of these genes and in order to elucidate its possible association with breast cancer risk we have looked for heterozygous mutations along its entire coding sequence and exon-intron boundaries in $95 \mathrm{BRCA} / 1$ 2-negative index cases from Spanish breast cancer families. We did not detect any pathogenic sequence change, which would indicate that $F A N C B$ does not confer a high risk of breast cancer or makes a major contribution to hereditary breast cancer. This is consistent with the overall lack of involvement of FA core complex genes in this type of cancer. However, larger-scale studies are required in order to rule out the existence of rare pathogenic $F A N C B$ alleles that might confer a modest effect. In this sense, a recent epidemiologic study assessing whether FA heterozygotes are at increased risk for cancer has shown evidences that FANCC mutations are possibly low-risk breast cancer susceptibility alleles [29], a major contribution of FANCC to familial breast cancer having been ruled out in a previous report [8].

Since the initial description of $P A L B 2$ as a breast cancer susceptibility gene [16], three more studies have been published that screened the gene in BRCA1/2-negative breast cancer families and/or women diagnosed with earlyonset breast cancer from different ethnic backgrounds such as Finish, French Canadian or Ashkenazy Jewish populations [17-19]. Founder mutations were found in Finish and, recently, in French Canadian population accounting for $1 \%$ of all breast cancer in Finland and about $0.5 \%$ of breast cancer cases affecting young French Canadian women [18, 19]. Data from these studies suggested that the relative risk for breast cancer associated with PALB2 mutations might be greater than 2, which has emphasized the need for further $P A L B 2$ analysis in different populations so that solid conclusions on the associated breast cancer relative risk can be established. Mutations in the gene seem to be individually rare, suggesting that there are not mutational hot-spots along its sequence and that the disease-alleles are heterogeneous. However, identification and characterization of more mutations is required. Similarly, attention has been drawn towards the identification of more PALB2 tumors in order to evaluate the existence of a $P A L B 2$-tumour distinctive phenotype.

Our search for $P A L B 2$ germline mutations in 95 probands from Spanish BRCA1/2-negative breast cancer families revealed only one pathogenic sequence variant accounting for a frequency of $1 \%$ in our series. Our results indicate that frequency of $P A L B 2$ mutations in Spanish hereditary breast cancer families is similar to that described in other populations where a founder effect was not observed [16]. The pathogenic change consisted of a nonpreviously described truncating mutation, c.1056_1057delGA (p.K353IfsX7). This mutation is the second most $5^{\prime}$ deleterious mutation described so far. In contrast to the most proximal one described by Tischkowitz et al. c.229delT (p.C77VfsX100) that generates a fusion protein consisting of 76 residues of the PALB2 native protein and a long nonnative tail of 99 residues [17], c.1056_1057delGA is predicted to generate a stop 7 codons downstream the first amino acid affected by the frameshift. Tischkowitz et al. suggested that such a premature truncation of the native protein might be related to the strong breast cancer history observed in the family harboring the c.229delT mutation and proposed that there might be a genotype-phenotype correlation. In this respect the pedigree of the family carrying c.1056_1057delGA (p.K353IfsX7) did not show particularly aggressive characteristics but it is worthnoting that it corresponded to a male and female breast cancer family. In a previous study, $6.7 \%(1 / 15)$ of such families carried PALB2 mutations compared to $1 \%$ (9/908) of families with female breast cancer only [16]. In our series the c.1056_1057delGA mutation affected to 1 out of 11 ( $9 \%$ ) families with male and female breast cancer. The small size of our series does not allow us to be more conclusive, but our results are consistent with the notion that PALB2 might confer a higher relative risk of breast cancer in men than in women, which seems plausible for a BRCA2-related gene.

Comprehensive screening of the previously described recurrent PALB2 mutations c.3113G $>$ A (p. W1038X), c.3116delA (p. N1039IfsX2) and c.3549C $>$ G (p.Y1183X) [16] in nearly $800 B R C A 1 / 2$-negative Spanish breast cancer families did not reveal any positive case. Similarly, extended analysis of the novel c.1056_1057delGA (p.K353IfsX7) mutation did not identify any other carrier of this change additional to that found in the initial set of 95 BRCA1/2-negative patients. These results confirm that, except for founder mutations, PALB2 alterations seem to be individually rare with no common or prevalent pathogenic alleles that might show different frequencies in different populations.

Analysis of the breast tumor from the c.1056_1057 delGA (p.K353IfsX7) mutation carrier determined the existence of $\mathrm{LOH}$. This is the first example of $\mathrm{LOH}$ occurring in a $P A L B 2$-associated tumor out of 11 analyzed to date $[17,18]$. Different mechanisms have been proposed to explain $P A L B 2$ functional inactivation such as haploinsufficiency or a dominant-negative effect of the truncated proteins [17, 18]. Here we present evidence that PALB2 loss of function might also conform to the inactivation 
model of a classic tumor-suppressor gene. Our PALB2 tumor did not express oestrogen and progesterone receptors, which is also in contrast with prior observations that suggested that $P A L B 2$-associated tumors share phenotypic characteristic with $B R C A 2$ tumors $[17,18]$. In this respect and in agreement with more recent findings [19] our data indicate that such observation might not be widely applicable to $P A L B 2$-tumors. This heterogeneity emphasizes the value of $P A L B 2$ mutation reports and the need for further characterization of $P A L B 2$-associated tumors and families. Accumulation of data in that respect will help to draw meaningful conclusions on genotypic-phenotypic correlations and to properly evaluate the indication of germinal analysis of this gene in a clinical context.

Acknowledgements We are grateful to Prof. Nazneen Rahman for kindly providing us with DNA samples from carriers of the previously described c. $3113 \mathrm{G}>\mathrm{A}$, c.3116delA and c. $3549 \mathrm{C}>\mathrm{G}$ PALB2 mutations used as positive controls in our screening; G. Ribas for valuable advise on DHPLC conditions and for helpful discussions; O. Díez and M. Baiget (Department of Genetics, La Santa Creu i Sant Pau Hospital) for their contribution in the ascertainment of part of the families included in the study; S.M. Rodríguez-Pinilla and M. Cañamero for histopathological revision of the PALB2 tumor and definition of areas for further macrodisecction; R.L. Milne for biostatistical support; S. Carilla for his contribution to this project; F. Fernández, C. Alonso and L. Moreno for excellent technical assistance. M.J. García is supported by the Centro de Investigación Biomédica en Red de Enfermedades Raras (CIBERER), Instituto de Salud Carlos III, Spain. This work was partially supported by the Fancogene Project (Fundación Genoma España) Pharmamar and the Spanish Network of Cancer (Grant RTICC 06/0020/0021).

\section{References}

1. Joenje H, Patel KJ (2001) The emerging genetic and molecular basis of Fanconi anaemia. Nat Rev Genet 2:446-457

2. Smogorzewska A, Matsuoka S, Vinciguerra P et al (2007) Identification of the FANCI protein, a monoubiquitinated FANCD2 paralog required for DNA repair. Cell 129:289-301

3. Mathew CG (2006) Fanconi anaemia genes and susceptibility to cancer. Oncogene 25:5875-5884

4. Whitney MA, Saito H, Jakobs PM et al (1993) A common mutation in the FACC gene causes Fanconi anaemia in Ashkenazi Jews. Nat Genet 4:202-205

5. Callen E, Casado JA, Tischkowitz MD et al (2005) A common founder mutation in FANCA underlies the world's highest prevalence of Fanconi anemia in Gypsy families from Spain. Blood 105:1946-1949

6. Wang W (2007) Emergence of a DNA-damage response network consisting of Fanconi anaemia and BRCA proteins. Nat Rev Genet 8:735-748

7. Howlett NG, Taniguchi T, Olson S et al (2002) Biallelic inactivation of BRCA2 in Fanconi anemia. Science 297:606-609

8. Seal S, Barfoot R, Jayatilake H et al (2003) Evaluation of Fanconi Anemia genes in familial breast cancer predisposition. Cancer Res 63:8596-8599

9. Luo L, Lei H, Du Q et al (2002) No mutations in the BACH1 gene in BRCA1 and BRCA2 negative breast-cancer families linked to 17q22. Int J Cancer 98:638-639
10. Karppinen SM, Vuosku J, Heikkinen K et al (2003) No evidence of involvement of germline BACH1 mutations in Finnish breast and ovarian cancer families. Eur J Cancer 39:366-371

11. Vahteristo P, Yliannala K, Tamminen A et al (2006) BACH1 Ser919Pro variant and breast cancer risk. BMC Cancer 6:19

12. Lewis AG, Flanagan J, Marsh A et al (2005) Mutation analysis of FANCD2, BRIP1/BACH1, LMO4 and SFN in familial breast cancer. Breast Cancer Res 7:R1005-1016

13. Seal S, Thompson D, Renwick A et al (2006) Truncating mutations in the Fanconi anemia J gene BRIP1 are low-penetrance breast cancer susceptibility alleles. Nat Genet 38:1239-1241

14. Xia B, Dorsman JC, Ameziane N et al (2007) Fanconi anemia is associated with a defect in the BRCA2 partner PALB2. Nat Genet 39:159-161

15. Reid S, Schindler D, Hanenberg $\mathrm{H}$ et al (2007) Biallelic mutations in PALB2 cause Fanconi anemia subtype FA-N and predispose to childhood cancer. Nat Genet 39:162-164

16. Rahman N, Seal S, Thompson D et al (2007) PALB2, which encodes a BRCA2-interacting protein, is a breast cancer susceptibility gene. Nat Genet 39:165-167

17. Tischkowitz M, Xia B, Sabbaghian N et al (2007) Analysis of PALB2/FANCN-associated breast cancer families. Proc Natl Acad Sci USA 104:6788-6793

18. Erkko H, Xia B, Nikkila J et al (2007) A recurrent mutation in PALB2 in Finnish cancer families. Nature 446:316-319

19. Foulkes WD, Ghadirian P, Akbari MR et al (2007) Identification of a novel truncating PALB2 mutation and analysis of its contribution to early-onset breast cancer in French-Canadian women. Breast Cancer Res. doi:10.1186/bcr1828. (in press)

20. Milne RL, Ribas G, Gonzalez-Neira A et al (2006) ERCC4 associated with breast cancer risk: a two-stage case-control study using high-throughput genotyping. Cancer Res 66:9420-9427

21. Llort G, Munoz CY, Tuser MP et al (2002) Low frequency of recurrent BRCA1 and BRCA2 mutations in Spain. Hum Mutat 19:307

22. Diez O, Osorio A, Duran M et al (2003) Analysis of BRCA1 and BRCA2 genes in Spanish breast/ovarian cancer patients: a high proportion of mutations unique to Spain and evidence of founder effects. Hum Mutat 22:301-312

23. Osorio A, Barroso A, Martinez B et al (2000) Molecular analysis of the BRCA1 and BRCA2 genes in 32 breast and/or ovarian cancer Spanish families. Br J Cancer 82:1266-1270

24. de la Hoya M, Perez-Segura P, Van Orsouw N et al (2001) Spanish family study on hereditary breast and/or ovarian cancer: analysis of the BRCA1 gene. Int J Cancer 91:137-140

25. Beristain E, Martinez-Bouzas C, Guerra I et al (2007) Differences in the frequency and distribution of BRCA1 and BRCA2 mutations in breast/ovarian cancer cases from the Basque country with respect to the Spanish population: implications for genetic counselling. Breast Cancer Res Treat 106:255-262

26. den Dunnen JT, Paalman MH (2003) Standardizing mutation nomenclature: why bother? Hum Mutat 22:181-182

27. den Dunnen JT, Antonarakis SE (2000) Mutation nomenclature extensions and suggestions to describe complex mutations: a discussion. Hum Mutat 15:7-12

28. Rahman N, Scott RH (2007) Cancer genes associated with phenotypes in monoallelic and biallelic mutation carriers: new lessons from old players. Hum Mol Genet 16 Spec No 1:R60R66

29. Berwick M, Satagopan JM, Ben-Porat L et al (2007) Genetic heterogeneity among Fanconi anemia heterozygotes and risk of cancer. Cancer Res 67:9591-9596 